Published in final edited form as:

Headache. 2006 June ; 46(Suppl 1): S3-S8.

\title{
Calcitonin Gene-Related Peptide (CGRP) and Migraine
}

\author{
Paul L. Durham, PhD \\ Department of Biology, Missouri State University, Springfield, MO.
}

\begin{abstract}
The neuropeptide calcitonin gene-related peptide (CGRP) has long been postulated to play an integral role in the pathophysiology of migraine. While clinical findings are consistent with such a role, the specific pathogenic mechanisms of CGRP in migraine have remained speculative until recently. Through advances in molecular neuroscience, the pathogenic mechanisms of CGRP in migraine have begun to be elucidated. This paper discusses the hypothesized role of CGRP in migraine and reviews recent findings on the molecular mechanisms of this neuropeptide in migraine pathophysiology. Studies in cultured trigeminal neurons demonstrate that CGRP is released from trigeminal ganglia cells, that CGRP transcription is increased under conditions mimicking neurogenic inflammation, that migraine pharmacotherapies can both reduce CGRP release and inhibit CGRP transcription, and that tumor necrosis factor- $\alpha$ (TNF- $\alpha$ ), an endogenous inflammatory mediator implicated in migraine, can stimulate CGRP transcription. Together, the results suggest that, in migraine, activation of trigeminal nerves release CGRP and other peptides that cause the release of proinflammatory mediators. These mediators further increase CGRP synthesis and release over hours to days in correspondence with the 4- to 72-hour duration of a typical migraine episode. The increased CGRP synthesis and release might be mediated by activation of mitogen-activated protein kinase pathways, which, in turn, can be modulated by endogenous inflammatory substances such as TNF- $\alpha$ and affected by drugs such as sumatriptan.
\end{abstract}

\section{Keywords}

inflammation; mitogen activated protein kinase; trigeminal; tumor necrosis factor alpha

The neuropeptide calcitonin gene-related peptide (CGRP) has long been postulated to play an integral role in the pathophysiology of migraine. ${ }^{1}$ While clinical findings are consistent with such a role, the specific pathogenic mechanisms of CGRP in migraine have remained speculative until recently. Through advances in molecular neuroscience, the pathogenic mechanisms of CGRP in migraine have begun to be elucidated. This paper discusses the hypothesized role of CGRP in migraine and reviews recent findings on the molecular mechanisms of this neuropeptide in migraine pathophysiology.

\section{HYPOTHESIZED PATHOPHYSIOLOGY OF MIGRAINE}

Migraine is a neurovascular disorder associated with dysfunction of the cerebral nerves and blood vessels. ${ }^{2,3}$ Although early theories posited the cerebral blood vessels as the site of origination of migraine attacks, current hypotheses place the primary dysfunction in the brain - probably in brainstem centers important in regulating vascular tone and pain sensation. In current hypotheses, migraine-specific triggers cause primary brain dysfunction,

(C) 2006 by American Headache Society Published by Blackwell Publishing

Address all correspondence to Dr. Paul L. Durham, Department of Biology, Missouri State University, 231 Temple Hall, Springfield, MO 65897. 
which causes dilation of cranial blood vessels that are innervated by sensory fibers of the trigeminal nerve to dilate. ${ }^{2,3}$ The dilated blood vessels mechanically activate perivascular trigeminal sensory nerve fibers. Activation of trigeminal sensory nerve fibers causes a pain response to be conveyed to the brainstem (and from there to higher brain centers) and evokes release of vasoactive peptides such as substance $P$ and CGRP from trigeminal fibers. These peptides exacerbate vasodilation and cause neurogenic inflammation characterized by vasodilation, leakage of blood vessels, and degranulation of mast cells. ${ }^{4}$ The vasodilation and neurogenic inflammation further increase activation of the sensory trigeminal fibers, perpetuate the release of vasoactive peptides including CGRP, and modulate transmission of pain impulses to the brain. As migraine progresses, the brainstem and spinal cord centers that are the first to receive the pain impulses from the trigeminal nerve are hypothesized to become sensitized with a resultant worsening of headache pain and increased sensitivity to environmental and other stimuli. ${ }^{3}$

\section{CGRP IN MIGRAINE}

CGRP is postulated to be involved in several of the pathophysiological processes, including dilation of cerebral and dural blood vessels, release of inflammatory mediators from mast cells, and transmission of nociceptive information from intracranial blood vessels to the nervous system. ${ }^{4}$ A 37 -amino-acid neuropeptide CGRP is the most potent known peptidergic dilator of peripheral and cerebral blood vessels. ${ }^{5,6}$ In the trigeminovascular system, cell bodies in the trigeminal ganglia constitute the main source of CGRP. Activation of the trigeminal nerve causes CGRP to be released from perivascular nerve endings.

Several findings in migraineurs support a role of CGRP in migraine. First, serum concentrations of CGRP are elevated during migraine attacks as well as episodes of other neurovascular headache types such as cluster headache. ${ }^{7-10}$ Furthermore, relief of migraine pain by triptans coincides with reduction in or normalization of CGRP concentrations in blood. ${ }^{9,11,12}$ The findings that selective CGRP receptor antagonists reduce vasodilation and neurogenic inflammation and confer clinical benefit in migraine further support an integral role of CGRP in migraine. ${ }^{4,13}$

\section{MOLECULAR NEUROSCIENCE OF CGRP AND MIGRAINE}

Studies involving cultured trigeminal neurons complement and extend these clinical observations. The results of these studies demonstrate that CGRP is released from trigeminal ganglia neurons, that CGRP transcription is increased under conditions mimicking neurogenic inflammation, that migraine pharmacotherapies can both reduce CGRP release and inhibit CGRP transcription, and that tumor necrosis factor- $\alpha$ (TNF- $\alpha$ ), an endogenous inflammatory mediator implicated in migraine, can stimulate CGRP transcription.

\section{CGRP RELEASE FROM TRIGEMINAL GANGLIA NEURONS UNDER CONDITIONS MIMICKING NEUROGENIC INFLAMMATION}

Neurogenic inflammation is hypothesized to enhance CGRP release in migraine. To explore this possibility in vitro, the effect of an inflammatory cock-tail including bradykinin, histamine, serotonin, and prostaglandin E2 - agents that mediate neurogenic inflammationon release of CGRP was assessed in cultured trigeminal neurons of Sprague-Dawley rats. ${ }^{14}$ In this preparation, which was specifically designed to assess CGRP release, primary cultures of rat trigeminal ganglia were enriched for neuronal cells to yield a culture containing nearly all CGRP-positive neurons. Treatment of the neurons with potassium chloride, which mimics the neuronal depolarization associated with trigeminal activation, caused a large increase in CGRP release relative to basal conditions (Fig. 1). ${ }^{14}$ Likewise, 
treatment of the neurons with capsaicin, which stimulates trigeminal sensory fibers, caused a marked increase in CGRP release (Fig. 1). Treatment of the neurons with the inflammatory cocktail, in an attempt to mimic neurogenic inflammation, was associated with an increase in CGRP release at least as large as that produced by potassium chloride or capsaicin (Fig. 1). The finding that trigeminal ganglia neurons release CGRP under conditions mimicking neurogenic inflammation is consistent with a role of CGRP in migraine.

\section{REGULATION OF CGRP RELEASE BY A TRIPTAN}

Treatment of migraine attacks with triptans, which are $5 \mathrm{HT}_{1 \mathrm{~B} / 1 \mathrm{D}}$ agonists, relieves pain, an effect that coincides with normalization of CGRP concentration in serum. ${ }^{7}$ These observations are consistent with the possibility that triptan-mediated inhibition of CGRP release from trigeminal neurons contributes to clinical efficacy. To assess whether triptans can directly inhibit release of CGRP from trigeminal neurons, the effect of sumatriptan on release of CGRP was assessed in cultured trigeminal neurons under basal conditions and under stimulated conditions simulating trigeminal activation during migraine. ${ }^{14}$ The results show that sumatriptan dose-dependently inhibited release of CGRP from trigeminal sensory neurons stimulated with potassium chloride. This effect of sumatriptan was blocked by administration of the $5 \mathrm{HT}_{1}$ receptor antagonist methiothepin. Release of CGRP was inhibited within 1 hour after sumatriptan treatment, a time period in the range of the reported onset of action of sumatriptan in migraineurs. The concentration of sumatriptan required for inhibition of CGRP release in culture was higher than the estimated plasma concentrations in patients administered the drug for migraine. Possibly, higher sumatriptan concentrations are required in vitro because of a low-effective receptor number, lack of colocalization of the receptors and the secretory apparatus at nerve endings, and/or refractoriness of chronically stimulated cultures.

Besides inhibiting potassium-stimulated release of CGRP, sumatriptan and two other $5 \mathrm{HT}_{1}$ receptor agonists, CGS 12066A and L-694,294, inhibited release of CGRP from trigeminal sensory neurons stimulated with the inflammatory cocktail. ${ }^{14}$ Sumatriptan did not affect CGRP release from unstimulated trigeminal sensory neurons. These observations demonstrate that $5 \mathrm{HT}_{1}$ agonists directly inhibit CGRP release from activated trigeminal neurons.

Additional experiments conducted to elucidate the signaling pathway by which sumatriptan inhibited CGRP release show that the inhibitory effect of sumatriptan was associated with a slow, prolonged elevation of intracellular calcium and activation of an okadaic acidsensitive phosphatase. ${ }^{14}$ The results are consonant with the possibility that sumatriptan inhibits CGRP release from trigeminal neurons by stimulating a sustained elevation in intracellular calcium and a consequent increase in phosphatase activity. Through these mechanisms, sumatriptan might interrupt a harmful feedback loop whereby CGRP release stimulates neurogenic inflammation that in turn stimulates additional CGRP release.

\section{REGULATION OF CGRP RELEASE BY BOTULINUM TOXIN TYPE A}

Research has recently been undertaken to determine whether or not other classes of migraine therapy, like triptans, inhibit CGRP release from trigeminal neurons. One series of experiments assessed the effects on CGRP release of botulinum toxin type A, which has been demonstrated to have prophylactic efficacy in migraine and, unlike triptans, is not a $5 \mathrm{HT}_{1}$ agonist. ${ }^{15}$ Release of CGRP from trigeminal sensory neurons was assessed in the culture preparation described above under unstimulated (basal) conditions and in response to stimulation by potassium chloride and capsaicin. The results show that botulinum toxin type A inhibited release of CGRP from trigeminal sensory neurons stimulated with potassium chloride or capsaicin. This inhibition was observed at botulinum toxin type A concentrations 
in culture that were within the range of the tissue concentrations achieved after local injection, the typical way of administering this migraine therapy clinically. Botulinum toxin type A did not affect CGRP release from unstimulated trigeminal sensory neurons. The results are consistent with the possibility that the prophylactic efficacy of botulinum toxin type A, like the acute efficacy of triptans, is partly attributed to its inhibitory effect on release of CGRP from trigeminal neurons.

\section{CGRP TRANSCRIPTION IN MIGRAINE}

The increased CGRP release observed shortly after initial stimulation of cultured trigeminal neurons and inhibition of this stimulated CGRP release by migraine medications might reflect cellular events occurring relatively early during the course of a migraine episode. That migraine attacks can last for up to 3 days suggests that a sustained increase in CGRP synthesis and release and sustained associated neurogenic inflammation can also occur. CGRP synthesis is controlled by a CGRP promoter that contains multiple regulatory sites including an upstream enhancer element. Mechanisms controlling the CGRP promoter were recently assessed in primary cultures of trigeminal neurons. ${ }^{16}$

\section{Effect of Activation of Trigeminal Neurons on CGRP Promoter Activity}

In 1 series of studies, the effect of activation of trigeminal neurons on CGRP promoter activity was investigated after treatment of trigeminal cultures either with potassium chloride to mimic depolarization or with the proinflammatory agent nerve growth factor, which is increased during cortical spreading depression. ${ }^{16}$ CGRP promoter activity was increased relative to untreated conditions after treatment with either potassium chloride or nerve growth factor. The increase in CGRP promoter activity reflects increased CGRP transcription (and synthesis). It was determined in other experiments that nerve growth factor activated the CGRP promoter by stimulating mitogen-activated protein kinases (MAPKs).

\section{Effect of $5 \mathrm{HT}_{1}$ Agonists on CGRP Promoter Activity}

Both nerve growth factor- and MAPK-stimulated CGRP promoter activities were found to be inhibited by sumatriptan. ${ }^{16}$ The regulation of CGRP promoter activity by sumatriptan was associated with a prolonged increase in intracellular calcium but was not linked to a cyclic AMP-dependent mechanism. The ionophore ionomycin, which produced an increase in calcium levels of similar amplitude and duration as that produced by sumatriptan, mimicked the action of sumatriptan on MAPK activity. These findings are consonant with the possibility that the sustained increase in intracellular calcium constitutes the mechanism by which sumatriptan regulates CGRP gene expression.

The ability of $5 \mathrm{HT}_{1}$ agonists other than sumatriptan to decrease CGRP promoter activity was confirmed in another series of studies that used an adenoviral vector to measure expression and regulation of the CGRP promoter in trigeminal neuronal cultures. ${ }^{17}$ Like sumatriptan, the $5 \mathrm{HT}_{1}$ agonists CGS 12066 , rizatriptan, and eletriptan decreased CGRP promoter activity by 2 to 3 times relative to that with vehicle control cultures (Fig. 2). ${ }^{17}$

\section{Endogenous Regulation of CGRP Transcription in Migraine}

Another series of investigations was undertaken to identify possible endogenous regulators of CGRP transcription in migraine. ${ }^{18}$ The cytokine TNF- $\alpha$ was studied in view of previous observations that it is among the most consistently elevated cytokine in migraine and that it, like nerve growth factor in the experiments described above, activates MAPK pathways in various cell types including neurons. ${ }^{19}$ The results of the investigations reveal that TNF- $\alpha-1$ receptors were present on most CGRP-containing rat trigeminal ganglia neurons. In 
addition, CGRP release from cultured trigeminal neurons was increased after treatment with TNF- $\alpha$ or ceramide, an intracellular signaling intermediate from the TNF- $\alpha-1$ receptor. Finally, TNF- $\alpha$ caused an increase in CGRP promoter activity and activated MAPK pathways. These findings provide evidence of a TNF- $\alpha$-induced proinflammatory signal cascade, including MAPKs, that increases the synthesis and release of CGRP in trigeminal ganglion neurons in migraine. The results suggest therapeutic potential of inhibitors of MAPK activity in the treatment of migraine and other inflammatory disorders involving trigeminal nerves.

\section{CONCLUSIONS}

This series of studies in cultured trigeminal neurons extends clinical observations supporting an integral role of CGRP in migraine and elucidates mechanisms by which CGRP may contribute to migraine pathophysiology. Together, these results suggest that activation of trigeminal nerves triggers release of CGRP and other peptides that cause the release of proinflammatory mediators. The mediators further increase CGRP synthesis and release over hours to days in correspondence with the 4- to 72-hour duration of a typical migraine episode (Fig. 3) ${ }^{14}$ The increased CGRP synthesis and release might be mediated by activation of MAPK pathways, which, in turn, can be modulated by endogenous inflammatory substances such as TNF- $\alpha$ and affected by drugs such as sumatriptan. The sustained elevation of intracellular calcium associated with administration of sumatriptan blocks the MAPK-mediated activation of CGRP gene expression. Given the importance of CGRP in migraine pathology, it will be imperative to have a better understanding of the cellular events that increase CGRP synthesis and release from trigeminal neurons. This knowledge will likely lead to the identification of novel molecular targets to more selectively modulate CGRP expression during migraine and other types of headache.

\section{REFERENCES}

1. Goadsby PJ, Edvinsson L, Ekman R. Release of vasoactive peptides in the extracerebral circulation of humans and the cat during activation of the trigeminovascular system. Ann Neurol. 1988; 23:193-196. [PubMed: 2454066]

2. Goadsby PJ, Lipton RB, Ferrari MD. Migraine-Current understanding and treatment. N Engl J Med. 2002; 346:257-270. [PubMed: 11807151]

3. Hargreaves RJ, Shepheard SL. Pathophysiology of migraine-new insights. Can J Neurol Sci. 1999; 26:S12-S19. [PubMed: 10563228]

4. Durham PL. CGRP receptor antagonists-A fresh approach to migraine therapy? N Engl J Med. 2004; 350:1073-1074. [PubMed: 15014178]

5. Brain SD, Williams TJ, Tippins JR, Morris H, MacIntyre I. Calcitonin gene-related peptide is a potent vasodilator. Nature. 1985; 313:54-56. [PubMed: 3917554]

6. McCulloch J, Uddman R, Kingman TA, Edvinsson L. Calcitonin gene-related peptide: Functional role in cerebrovascular regulation. Proc Natl Acad Sci USA. 1986; 83:5731-5735. [PubMed: 3488550]

7. Goadsby PJ, Edvinsson L. The trigeminovascular system and migraine: Studies characterizing cerebrovascular and neuropeptide changes seen in humans and cats. Ann Neurol. 1993; 33:48-53. [PubMed: 8388188]

8. Goadsby PJ, Edvinsson L. Human in vivo evidence for trigeminovascular activation in cluster headache. Neuropeptide changes and effects of acute attack therapies. Brain. 1994; 117:427-434. [PubMed: 7518321]

9. Edvinsson L, Goadsby PJ. Neuropeptides in migraine and cluster headache. Cephalalgia. 1994; 14:320-327. [PubMed: 7828188] 
10. Fanciullacci M, Alessandri M, Figini M, Geppetti P, Michelacci S. Increase in plasma calcitonin gene-related peptide from the extracerebral circulation during nitroglycerine-induced cluster headache attack. Pain. 1995; 60:119-123. [PubMed: 7540279]

11. Ferrari MD, Saxena PR. On serotonin and migraine: Aclinical and pharmacological review. Cephalalgia. 1993; 13:151-165. [PubMed: 8395342]

12. Buzzi MG, Bonamini M, Moskowitz MA. Neurogenic model of migraine. Cephalalgia. 1995; 15:277-280. [PubMed: 7585923]

13. Durham PL. CGRP receptor antagonist: A new choice for acute treatment of migraine? Curr Opin Investig Drugs. 2004; 5:731-735.

14. Durham PL, Russo AF. Regulation of calcitonin gene-related peptide secretion by a serotonergic antimigraine drug. J Neurosci. 1999; 19:3423-3429. [PubMed: 10212302]

15. Durham PL, Cady R, Cady R. Regulation of calcitonin gene-related peptide secretion from trigeminal nerve cells by botulinum toxin type A: Implications for migraine therapy. Headache. 2004; 44:35-43. [PubMed: 14979881]

16. Durham PL, Russo AF. Stimulation of the calcitonin gene-related peptide enhancer by mitogenactivated protein kinases and repression by an antimigraine drug in trigeminal ganglia neurons. J Neurosci. 2003; 23:807-815. [PubMed: 12574409]

17. Durham PL, Dong PX, Belasco KT, Kasperski J, Gierasch W, Edvinsson L, et al. Neuronal expression and regulation of CGRP promoter activity following viral gene transfer into cultured trigeminal ganglia neurons. Brain Res. 2004; 997:103-110. [PubMed: 14715155]

18. Bowen EJ, Schmidt TW, Firm CS, Russo A, Durham P. Tumor necrosis factor- $\alpha$ stimulation of calcitonin gene-related peptide expression and secretion from rat trigeminal ganglion neurons. $\mathrm{J}$ Neurochem. 2006; 96:65-77. [PubMed: 16277606]

19. Schafers M, Svensson C, Sommer C, Sorkin L. Tumor necrosis factor-alpha induces mechanical allodynia after spinal nerve ligation by activation of p38 MAPK in primary sensory neurons. $\mathrm{J}$ Neurosci. 2003; 23:2517-2521. [PubMed: 12684435] 


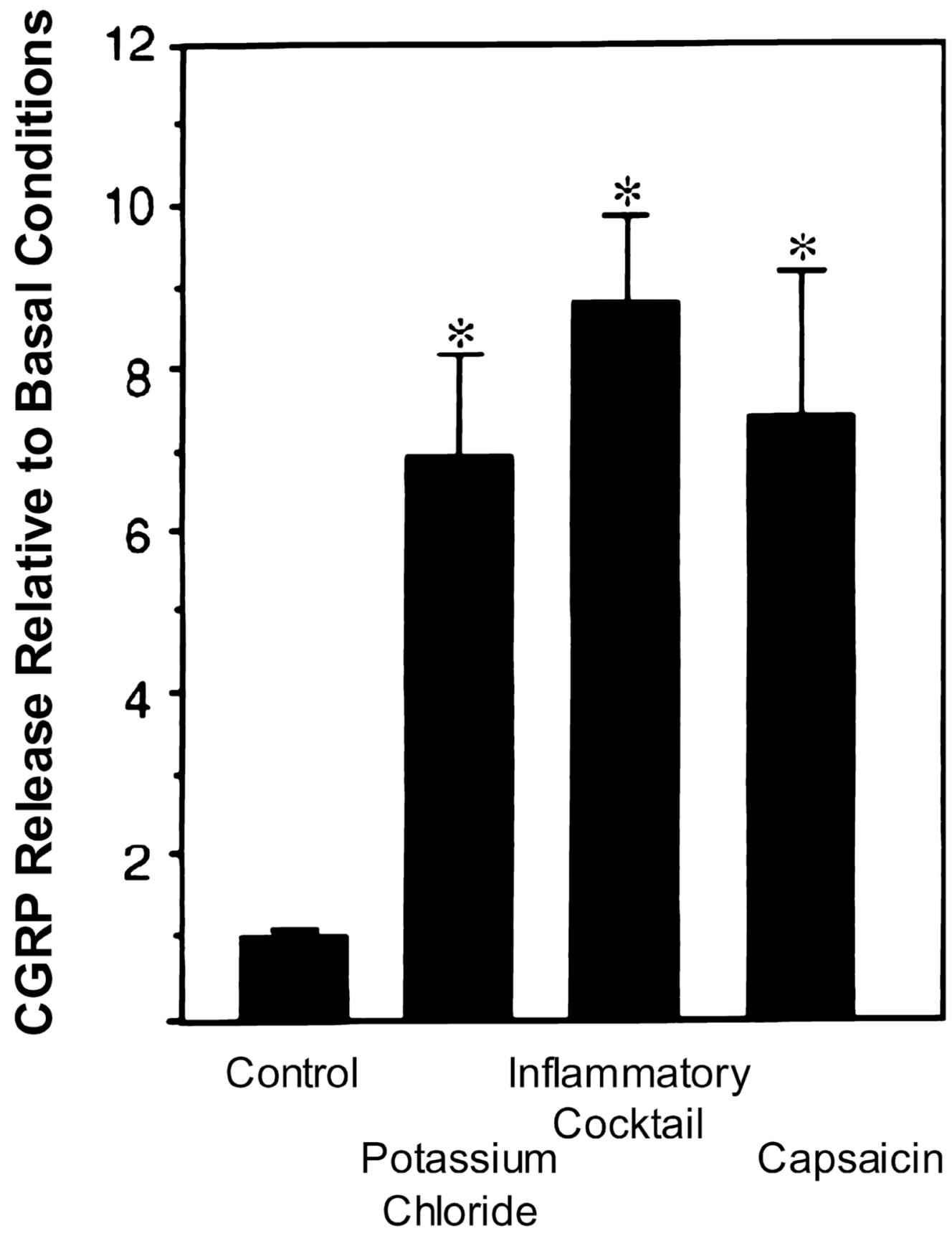

Fig 1.

Expression and release of CGRP from cultured trigeminal neurons. ${ }^{14}$ The relative amount of CGRP released in 1 hour from untreated control cells or cells treated with $60 \mathrm{mM}$ potassium chloride, a cocktail of inflammatory agents, or $10 \mu \mathrm{M}$ capsaicin. The mean basal rate of CGRP release was $148 \pm 5 \mathrm{pg} / \mathrm{hour} / \mathrm{dish}(\mathrm{n}=36)$. ${ }^{*} P<.001$ compared with control.

(Reproduced with permission from Durham PL, Russo AF. Regulation of calcitonin generelated peptide secretion by a serotonergic antimigraine drug. J Neurosci. 1999;19:34233429). 


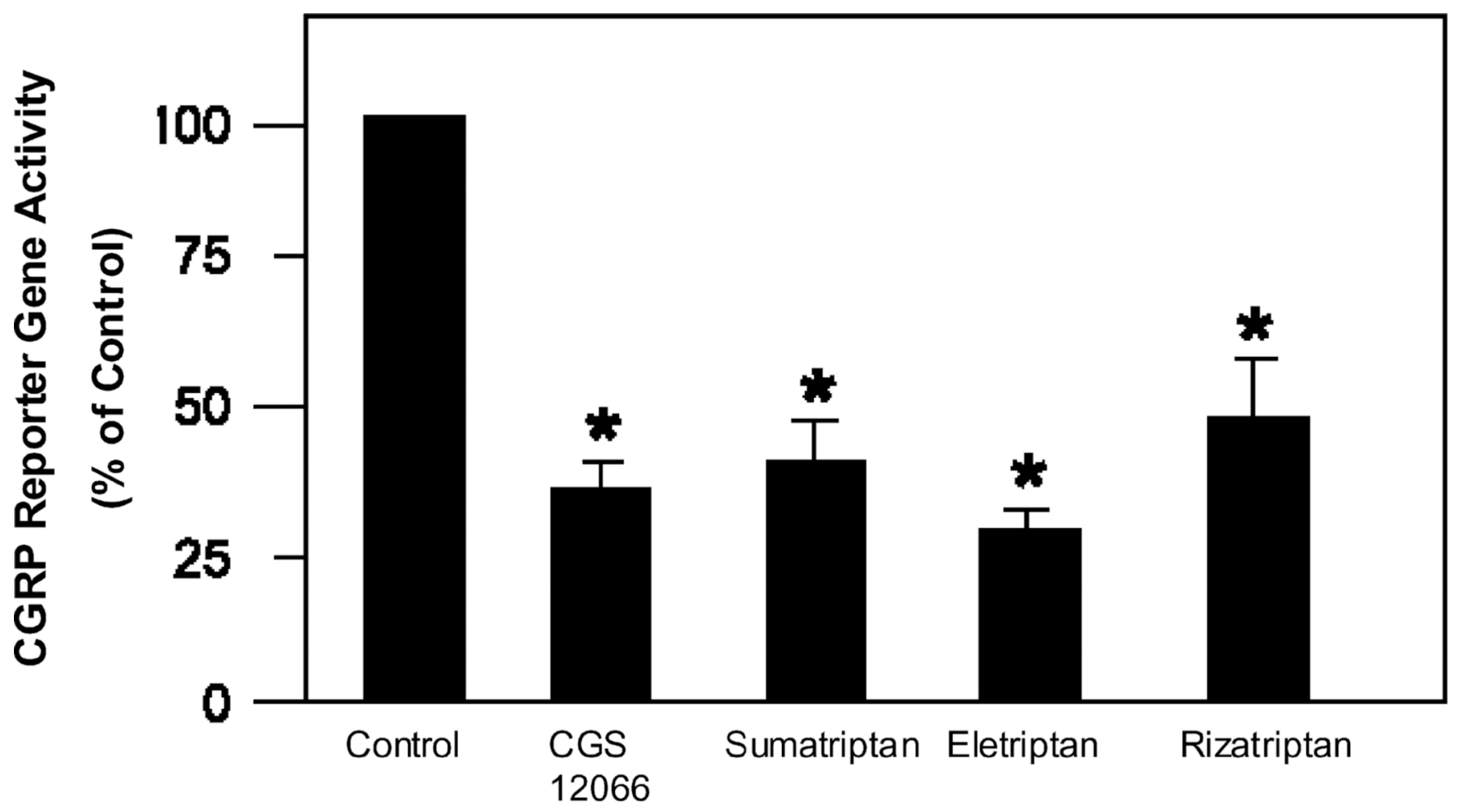

Fig 2.

Repression of CGRP promoter activity (expressed as \% of control CGRP reporter gene activity) in cultured trigeminal neurons. ${ }^{17}$ CGS 12066A, sumatriptan, eletriptan, and rizatriptan administered at $5 \mu \mathrm{M}$ for 18 hours repressed reporter gene activity relative to control values. ${ }^{*} P<.05$ compared with control. (Reproduced with permission from Durham PL, Dong PX, Belasco KT, et al. Neuronal expression and regulation of CGRP promoter activity following viral gene transfer into cultured trigeminal ganglia neurons. Brain Res. 2004;997:103-110). 
CNS

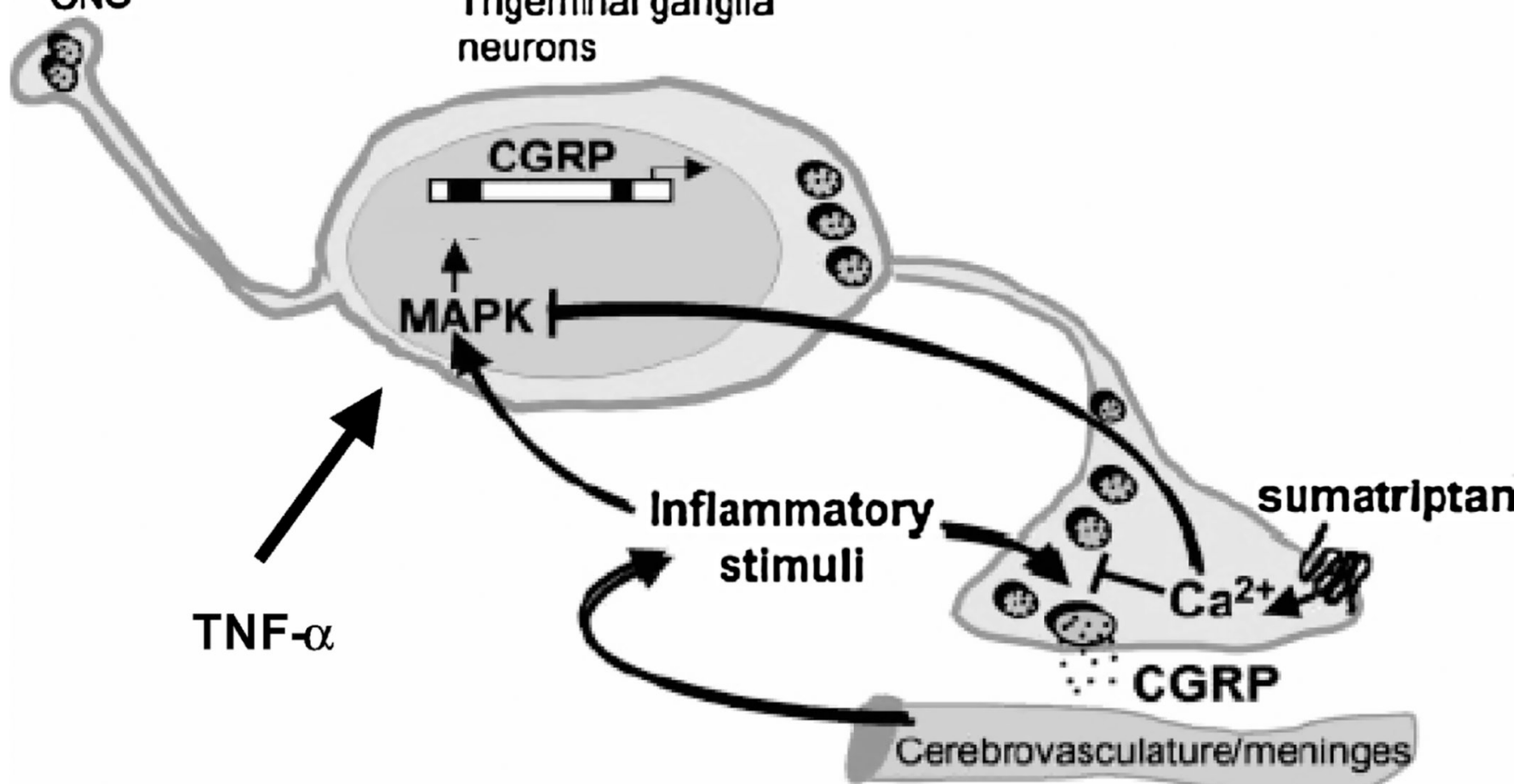

Fig 3.

CGRP regulation in trigeminal ganglia neurons. ${ }^{14}$ Activation of trigeminal nerves causes initial release of CGRP and other neuropeptides that promote release of inflammatory mediators. The inflammatory mediators, including TNF- $\alpha$, further increase CGRP synthesis and release via MAPKs. Sumatriptan can block MAPK activation by causing sustained elevation of intracellular calcium. CNS = central nervous system. (Reproduced with permission from Durham PL, Russo AF. Regulation of calcitonin gene-related peptide secretion by a serotonergic antimigraine drug. J Neurosci. 1999;19:3423-3429). 